\title{
Mental health in South East London general hospitals: using electronic patient records to explore associations between psychiatric diagnoses and length of stay in a patient cohort receiving liaison psychiatry input
}

\author{
Abbeygail Jones, Helen Todman and Mujtaba Husain
}

\section{Background}

Psychiatric illnesses are prevalent in general hospitals and associated with length of stay (LOS). Liaison psychiatry teams provide psychiatric care in acute hospitals and can improve mental health-related outcomes but, to achieve ambitious policy targets, services must understand local need.

\section{Aims}

Using electronic patient records, we investigate associations between psychiatric diagnoses and LOS in South East London hospitals.

\section{Method}

Patient records were extracted using the South London and Maudsley NHS Foundation Trust Biomedical Research Centre Case Register Interactive Search system. There were 6378 admissions seen by liaison psychiatry aged $<65$ years between 2011 and 2016. Linear mixed-effects models investigated the impact of psychiatric diagnoses on LOS. Potential confounders included medical diagnoses, gender, age, ethnicity, social deprivation, hospital site and investment per admission.

\section{Results}

According to marginal means, longer LOS is associated with primary diagnoses of organic disorders (mean: 23 days, $95 \% \mathrm{Cl}$ 20.39-25.61), depressive disorders (mean: 11.03 days, 95\% Cl 9.74-25.61) and psychotic disorders (mean: 10.63 days, 95\% Cl 8.75-12.51). Shorter LOS is associated with personality disorders (mean: 6.28 days, 95\% $\mathrm{Cl} 4.12-8.45$ ), bipolar affective disorders (mean 6.81 days, 95\% $\mathrm{Cl} 3.49-10.14$ ) and substance-related problems (mean 7.53 days, $95 \% \mathrm{Cl} 6.01-9.05$ ).

\section{Conclusions}

Psychiatric diagnoses have differential associations with inpatient LOS. Liaison psychiatry teams aim to mitigate the impact of psychiatric illness on patient and hospital outcomes but understanding local need and the wider context of care provision is needed to maximise potential benefits.

\section{Declaration of interest}

M.H. is a consultant liaison psychiatrist for King's College Hospital adult liaison psychiatry team. At the time of writing, H.T. was senior business manager at SLaM psychological medicine and integrated care clinical academic group. These may be considered financial and/or non-financial interests given the implications of findings for service funding.

\section{Keywords}

Liaison psychiatry; length of stay; mental health diagnoses; patient records.

\section{Copyright and usage}

(c) The Author(s) 2019. This is an Open Access article, distributed under the terms of the Creative Commons AttributionNonCommercial-NoDerivatives licence (http://creativecommons.org/licenses/by-nc-nd/4.0/), which permits non-commercial re-use, distribution, and reproduction in any medium, provided the original work is unaltered and is properly cited. The written permission of Cambridge University Press must be obtained for commercial re-use or in order to create a derivative work.

\section{Background}

The number of patients with comorbid physical and mental health problems is prevalent and rising, with implications for patient outcomes and healthcare provision. ${ }^{1,2}$ Comorbidity is associated with greater healthcare use, reduced life expectancy, reduced functionality and increased symptom burden. ${ }^{3,4}$ Subsequently, the National Health Service is challenged to treat patients with complex, chronic conditions as national policy targets aim to integrate mental and physical health. ${ }^{5,6}$ Psychiatric diagnoses are associated with longer length of stay (LOS) in general medical hospitals, independent of physical illness and demographic variables such as age and socioeconomic status. ${ }^{7-10}$ Longer LOS is associated with poorer functional outcomes for trauma patients, and medically fit patients waiting for discharge have greater risk of infection and mortality in the year post-discharge. ${ }^{11,12}$ By contributing to high bed occupancy, LOS is associated with adverse events, in-patient mortality, readmission and extended waiting times. ${ }^{13}$ Thus, identifying patients at risk of longer LOS has important clinical implications.

\section{Liaison psychiatry services}

Liaison psychiatry services are multidisciplinary teams providing psychiatric care and training for medical staff in acute hospitals. Four main liaison psychiatry service models are described (Core, Core24, Enchanced24 and Comprehensive); all models aim to improve care pathways and reduce costs by providing expertise in mental health, with higher-level models offering 24/7 availability, more specialist or regional services. ${ }^{14}$ Liaison psychiatry services are associated with reduced admissions and LOS as they facilitate the complex inpatient care and discharge planning needed by patients with mental illness. ${ }^{15,16}$ Following the Five Year Forward View, ${ }^{17}$ liaison psychiatry services are undergoing substantial changes that require an understanding of local needs to achieve key outcome measures such as reduced LOS. ${ }^{18}$ However, policy documents do not recommend how services might gain insight into local need. An ongoing liaison psychiatry collaborative evaluation ${ }^{19}$ recommend service audits may be used to identify 'resource intensive' patient groups that can then inform ongoing service development. 


\section{Identifying associations between different psychiatric diagnoses and LOS}

With aims to reduce LOS, release resources and improve quality of care, South London and Maudsley (SLaM) NHS Foundation Trust are implementing Core 24 liaison psychiatry services -24 h, 7 day a week liaison psychiatry services with rapid response in accident and emergency (A\&E) and wards across four hospital sites. We aimed to investigate associations between different psychiatric diagnoses and LOS across SLaM acute hospital sites, controlling for potential patient- and service-level confounders (physical health diagnoses, age, gender, social deprivation and investment per admission). At a local level, results will provide insight into the patients seen by liaison psychiatry in SLaM services but more broadly this paper provides an example of how patient records can be analysed to guide service development when implementing national policy.

\section{Method}

\section{Study design and setting}

This is a retrospective, observational study using hospital episode statistics (HES) and electronic patient records from SLaM NHS Foundation Trust. SLaM provides mental health services across South London boroughs (Southwark, Lambeth, Lewisham and Croydon). Hospitals within the trust provide specialist care to patients across the country.

\section{Data source}

Data was extracted from the SLaM Biomedical Research Centre Case Register Interactive Search (CRIS) system. Developed in 2008, CRIS provides access to de-identified electronic health records linked with HES from SLaM hospitals. There are approximately 250000 patient records and 300000 mental health cases recorded on the CRIS database $\mathrm{e}^{20}$ and it is approved as a secondary data resource by the National Research Ethics Committee South Central Oxford C (reference: 08/H0606/71+5). This study received CRIS approval in September 2017 (Project number 17-092). In May 2018, we extracted data for adult in-patient admissions to SLaM acute hospitals between 2007 and 2016 that had a liaison psychiatry episode occurring between hospital admission and discharge dates.

\section{Data selection}

Each row of data represented one admission episode. Only admissions at Croydon University Hospital, King's College Hospital $(\mathrm{KCH})$, Lewisham Hospital or St Thomas' Hospital liaison psychiatry were included as these sites are implementing Core24 liaison psychiatry services. Patients aged over 65 years were excluded because psychiatry teams for this age group have been developed separately, older adults present unique challenges to discharge relating to social factors, ${ }^{21}$ and the prominence of dementia and depression diagnoses in older adults could mask the effects of other diagnoses in working-age patients. ${ }^{22}$ Episodes without ICD- $10^{23}$ mental and behavioural disorder diagnoses or that could not be categorised were excluded. In total, 16218 episodes met inclusion criteria: admission on or after 1 April 2010, $<65$ years at admission, categorisable psychiatric diagnosis and referral to the Core24 liaison psychiatry team during admission (supplementary Fig. 1 available at https://doi.org/10.1192/bjo.2019.79). Liaison psychiatry referrals could be received from $\mathrm{A} \& \mathrm{E}$ or in-patient wards. There were no exclusion criteria relating to physical health diagnoses.

\section{Outcome}

LOS was calculated by subtracting admission date from discharge date. Where multiple episodes had the same patient identification and admission date, the latest available discharge date was used.

\section{Main predictor}

Psychiatric diagnoses were recorded according to ICD-10 categories in the Electronic Patient Journey System used by liaison psychiatry. Per admission, patients received a primary diagnosis and $<6$ secondary diagnoses. If the primary diagnosis could not be categorised, the first categorisable secondary diagnosis was used. As the sample were a liaison psychiatry patient cohort, psychiatric diagnoses were likely made or informed by a mental health professional (psychiatric nurse, core trainee or consultant) with more expertise in psychiatric diagnoses than other medical clinicians. Diagnoses were initially summarised into 12 categories (supplementary Table 1) with reference to the emergency care data-set, ${ }^{24}$ which provided a convenient model for grouping diagnoses accepted across hospital sites.

As a result of low observations, somatisation disorders, selfharm, eating disorders, dissociative and developmental disorders were excluded, leaving seven categories for analysis: anxiety disorders; depressive disorders; bipolar affective disorders; organic disorders (referring to dementia, delirium and other organic disorders); psychotic disorders; personality disorders and substance-related problems.

\section{Covariates}

Covariates were medical diagnoses, age, gender, ethnicity, social deprivation, liaison hospital site and investment per admission. In HES, medical diagnoses belong to one of 21 ICD-10 chapters. The first recorded diagnosis chapter informed the medical diagnosis variable, considered a proxy of the reason for admission. Age in years and gender (male/female) were recorded at admission. All episodes had a value indicating patient ethnicity. Eighteen categories for ethnicity were available: African (Black or Black British); any other Asian background; any other Black background; any other mixed background; any other White background; any other ethnic group; Bangladeshi (Asian or Asian British); British (White); Caribbean (Black or Black British); Chinese; Indian (Asian or Asian British); Irish (White); not known; not stated; Pakistani (Asian or Asian British); White and Asian (mixed); White and Black African (mixed); White and Black Caribbean (mixed). These were collapsed into a composite variable: any White ethnicity; any Black ethnicity; any Asian ethnicity; any mixed ethnicity; any other ethnicity; and not known.

Social deprivation was based on the Index of Multiple Deprivation (IMD) overall rank. The IMD provides value between 1 (most deprived) and 32482 (least deprived), calculated from weighted scores across seven domains: income, employment, health deprivation and disability, education, skills and training, barriers to housing and services, crime and living environment. A categorical variable indicated the hospital site of the episode. Liaison team names change over time and have recently been retrospectively altered in patient records. Data extraction provided the first liaison psychiatry team seen within an admission. Episodes were available from liaison psychiatry in-patient teams at all sites. There were no episodes from $\mathrm{KCH}$ A\&E liaison in the data-set. Liaison team cost per financial year was provided by the SLaM NHS Foundation Trust clinical academic group and use was approved by the quality subcommittee. An investment per admission variable was calculated by dividing yearly cost of a liaison service by number of admissions seen by the team in a given financial year. 


\section{Analyses}

Data preparation

Analyses were complete in Stata software. ${ }^{25,26}$ There were 9839 duplicates resulting from erroneous data entry and complications of data extraction. Duplicates were removed, leaving 6379 unique combinations of patient identification, admission date and psychiatric diagnosis. Following consultation with clinicians, an outlier with LOS equal to 991 days was dropped. There were instances where patients had multiple primary psychiatric diagnosis recorded for one admission. With no reliable method for determining which diagnosis was more valid, these duplicates were included $(n=128$, $2.0 \%$ sample). There were 6378 admissions from 5224 patients for analysis (supplementary Fig. 1).

\section{Inferential analysis}

Linear mixed-effects models examined the association between psychiatric diagnoses and LOS. Informed by previous literature, ${ }^{7}$ depressive disorders were set as the reference category/intercept. It was recognised that LOS could be affected by patient-level confounding factors not available for analysis (for example homelessness or safeguarding issues). Mixed-effects models accounted for this and repeated observations per patient, which violates independent observations assumptions of ordinary linear regression models, by specifying patient identification as a random effect. ${ }^{27}$ Predictor and confounder variables were set as fixed effects, defined as variables 'whose levels are of interest in their own right'. ${ }^{28}$

An initial linear mixed-effects model examined the crude association between psychiatric diagnoses and LOS. A 'full model' included all potential confounding variables. Models were run using the Stata command mixed, producing a coefficient, standard error, $Z$-statistic, $P$-value and $95 \% \mathrm{CIs}$ for each continuous variable or level of categorical variables. The sample size exceeded 30, meaning it was appropriate to retain the $Z$-statistic. ${ }^{29}$ Stata fit models using maximum likelihood theory, allowing comparison of models with different fixed effects. ${ }^{30}$

\section{Results}

\section{Participant characteristics}

A total of 6378 episodes were available after exclusion criteria were applied and data preparation was complete (supplementary Fig. 1). The distribution of episodes across each site was: $1113(17.45 \%)$ admissions from Croydon University Hospital, 1321 (20.71\%) from KCH, 1322 (20.73\%) from Lewisham Hospital and 2622 (41.11\%) from St Thomas' Hospital. There were 3226 (50.58\%) admissions of female patients and 3152 (49.42\%) were male patients. Average age was 40.53 years (s.d. $=12.83$, minimum 18.04, maximum 64.98). Episodes were identified by ethnicity as follows: 3954 (61.99\%) any White ethnicity; 984 (15.43\%) any Black; 250 (3.92\%) any Asian; 124 (1.94\%) any mixed; 369 (5.79\%) other; and 697 (10.93\%) unknown.

The psychiatric diagnoses seen each year varied between teams (supplementary Table 2). Across teams, depression and psychotic disorder diagnoses are increasing over time. LOS ranged from 0 to 396 days, $56.07 \%(n=3576)$ episodes had admissions lasting 0 or 1 day. Median LOS was 1 day and mean LOS was 10.06 days (s.d. = 27.54, minimum 0, maximum 396). Average LOS according to psychiatric diagnosis is reported in Table 1 and Fig. 1. There were 6046 (94.79\%) episodes (from 4984 patients) that had complete data for inferential analysis.

LOS had a strong positive skew and non-normal distribution requiring non-parametric tests. The skew is because of the contribution of short episodes from A\&E liaison psychiatry teams. LOS correlated with age $(r s=0.295, P<0.001)$ and investment per admission ( $r s=0.280, P<0.001)$ to a statistically significant level but not social deprivation $(r s=0.011, P=0.41)$. According to KruskalWallis tests of variance, LOS differed between psychiatric diagnoses $(H=193.78, \quad P<0.001)$, patient ethnicity $(H=48.13, P<0.001)$, medical diagnosis chapter $(H=2178.05, P<0.001)$ and in-patient liaison teams $(H=1647.93, \quad P<0.001)$. A Wilcoxon-MannWhitney test showed LOS differed between male and female patients $(Z=3.246, P=0.001)$.

\section{Linear mixed-effects models}

\section{crude association}

The crude model investigated the association between psychiatric diagnoses and LOS. Parameter estimates are displayed in Table 2. Fixed-effect estimates indicate depressive disorders and organic disorders are associated with longer LOS. Depressive disorders contributed on average 11 days to hospital LOS $(\beta=11.028, P<0.001$, 95\% CI 9.737-12.320). Organic disorders contributed an additional 12 days $(\beta=11.972, P<0.001,95 \%$ CI 9.099-14.845), indicating a mean in-patient admission of 23 days for patients with this category of diagnoses. In contrast, bipolar affective disorders, personality disorders and substance-related problems are associated with lower LOS than depressive disorders (Table 2). Random-effect estimates suggest variability in LOS is accounted for less by fixed-effect estimates than other patient-level variables not included in the crude model. This is demonstrated by the individual variance estimate exceeding the residual variance estimate.

\section{Full model}

The full-linear mixed-effects model (Table 3 and supplementary Table 3) contained all potential confounders of LOS. Depressive disorders remain associated with longer LOS. Bipolar affective or substance-related disorders are associated with shorter LOS, approximately 5 days and 3 days less, respectively. Anxiety disorders have a small effect, increasing LOS by less than a full day $(\beta=0.786$, s.e. $=0.958,95 \% \mathrm{CI}-1.091$ to $2.664, P=0.041)$. Presence of dementia, delirium or organic disorders is associated with approximately 7 days longer in hospital.

Greater age is a statistically significant predictor of longer LOS $(\beta=0.212$, s.e. $=0.275,95 \%$ CI $0.158-0.266, P<0.001)$. Lower IMD rank score, indicating greater social deprivation, is associated with shorter LOS to a statistically significant level $(\beta=-0.098$, s.e. $=$ $0.029,95 \%$ CI -0.156 to $0.041, P=0.041)$ KCH, Lewisham and St Thomas' liaison teams are associated with longer LOS using Croydon as the reference category (supplementary Table 3). Investment per admission has a statistically significant but objectively very small association with LOS. Ethnicity and gender are not associated with LOS in this study. A post-estimation test using the contrast command was run to determine if the joint effects of medical diagnoses on LOS are statistically different from zero. The combined effect of medical diagnosis chapters on LOS was statistically significant $\left(\chi^{2}(18)=679.33, P<0.001\right)$. For the aims of this study, the contribution or direction of effects for different medical diagnoses, or interactions with psychiatric diagnoses, was not estimated; instead, medical diagnoses acted as a proxy for physical health to determine if this had an impact on the association between psychiatric diagnoses and LOS.

Examining random effects from the full model reveals a smaller individual variance estimate (294.5233) compared with the crude model (426.975). This suggests the full model, containing all confounders as fixed effects, captures more of the variability in LOS than the crude model. However, the individual variance estimate is still large indicating that patient-level differences not measured 


\begin{tabular}{|c|c|c|c|c|c|c|}
\hline Mental health diagnosis category & Mean & Median & Minimum & Maximum & s.e. & $95 \% \mathrm{Cl}$ \\
\hline Dementia, delirium and organic & 26.03 & 8 & 0 & 337 & 2.35 & $21.43-30.62$ \\
\hline Depressive disorders & 11.97 & 1 & 0 & 344 & 0.77 & $10.47-13.47$ \\
\hline Psychotic disorders and schizophrenia & 10.66 & 2 & 0 & 396 & 0.93 & $8.84-12.49$ \\
\hline Anxiety disorders & 10.00 & 1 & 0 & 320 & 0.86 & $8.31-11.68$ \\
\hline Bipolar affective disorders & 7.84 & 1 & 0 & 181 & 1.18 & $5.52-10.15$ \\
\hline Substance related & 6.20 & 1 & 0 & 352 & 0.49 & $5.23-7.16$ \\
\hline Personality disorders & 4.64 & 1 & 0 & 198 & 0.55 & $3.57-5.71$ \\
\hline
\end{tabular}

or accounted for in our analysis, make a substantial contribution to LOS.

The contribution of the individual random effect was confirmed with a post-estimation interclass correlation (ICC) test using the estat icc command. This test represents the correlation of variables within individuals and the proportion of variance accounted for by the random effect. The ICC test can therefore evaluate the choice of a mixed-effects model. ICC for the crude model was 0.606 (s.e. $=$ 0.015 , 95\% CI 0.576-0.635) compared with 0.521 (s.e. $=0.017$, 95\% CI 0.489-0.554) for the full model. Thus, random effects at the patient-level account for approximately $60 \%$ variance in LOS in the crude model and $52 \%$ variance in LOS in the full model. These proportions are substantial and support the choice of mixed-effects models to account for patient-level variability.

\section{Relative contribution of psychiatric diagnoses}

Estimated marginal means were computed based on full-model estimates (Table 4). Marginal means provide a predicted LOS, extrapolated from average fixed-effect estimates, including estimates for the reference category. Marginal means suggest organic disorders, depressive disorders and psychotic disorders and schizophrenia are associated with longest LOS. Contrastingly, personality and bipolar affective disorders are predicted to have the shortest LOS.

\section{Model comparison}

Models were compared using a likelihood-ratio test. This method constrains parameters of confounders in the full model to zero in order to observe their influence on overall model fit. Results confirmed that the fit of the full model was better than the crude model to a statistically significant level $\left(\chi^{2}(30)=1172.42, P<0.001\right)-$ the combined addition of confounder variables makes a statistically significant improvement to model fit.

\section{Discussion}

This paper investigated associations between psychiatric diagnoses and LOS in acute hospitals. Linear mixed models show depressive disorders, anxiety disorders and organic disorders are associated with longer LOS whereas bipolar affective and substance-related disorders are associated with shorter LOS. Marginal means predict organic disorders have the longest LOS (23 days) followed by depressive disorders (11 days) and psychotic disorders and schizophrenia (10.6 days) whereas bipolar affective and personality disorders have the shortest. Older age, greater social deprivation, medical diagnoses, hospital site and investment per admission were statistically significant confounders of LOS.

Concordant with previous research, ${ }^{8,10}$ depression diagnoses and dementia are associated with longer LOS when controlling for medical illness and sociodemographics. The association between longer LOS and organic disorders in working-age adults may reflect the severity of these conditions in younger age groups. Furthermore, teams working with working-age adults may be less specialised for organic disorders such as dementia or delirium leading to longer LOS. In contrast to studies comparing patients with psychiatric diagnoses to those without, ${ }^{10}$ we report substance-related disorders are associated with shorter LOS. Substance-related diagnoses may be associated with longer LOS compared with no psychiatric diagnosis, but shorter LOS compared with other psychiatric conditions. Associations between LOS and

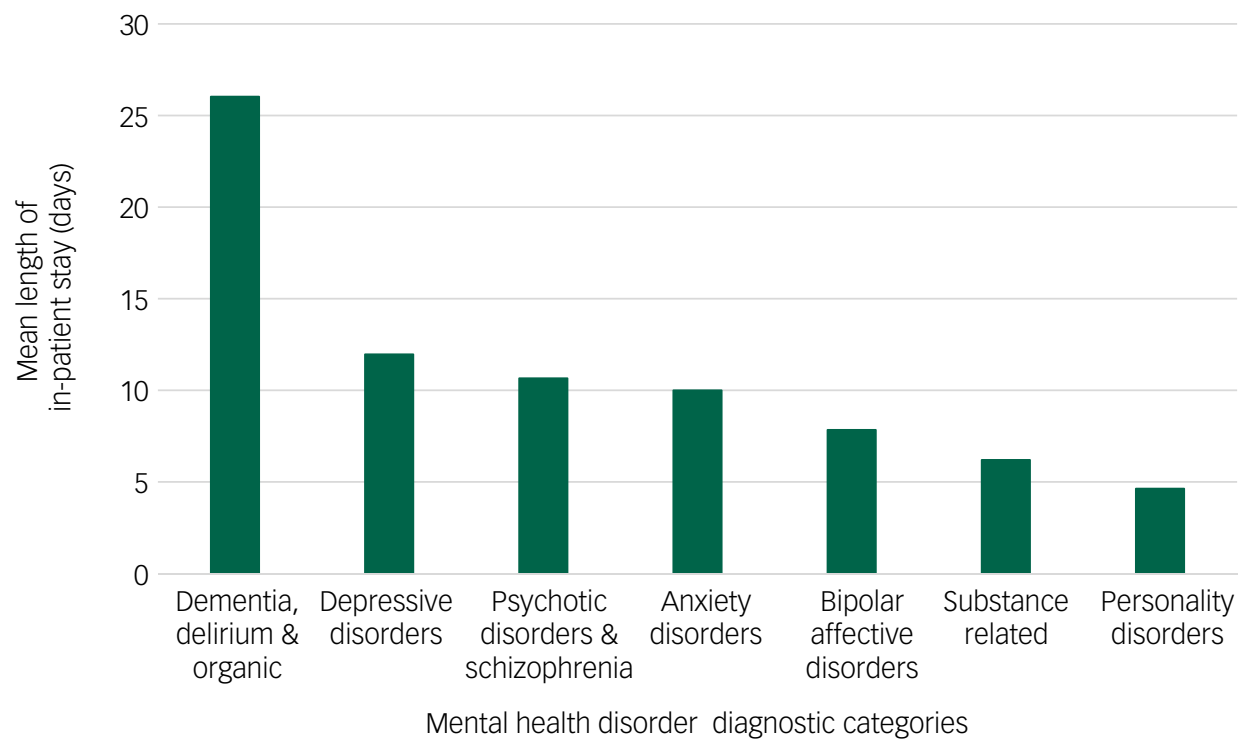

Fig. 1 Bar chart of mean length of stay (days) by mental health diagnosis category. 


\begin{tabular}{|c|c|c|c|c|c|c|}
\hline & Estimate & Variance & s.e. & Z-statistic & $P$ & $95 \% \mathrm{Cl}$ \\
\hline \multicolumn{7}{|l|}{ Fixed effects } \\
\hline \multicolumn{7}{|l|}{ Mental health diagnosis category ${ }^{a}$} \\
\hline Intercept ${ }^{a}$ & 11.028 & - & 0.659 & 16.74 & $<0.001$ & 9.737 to 12.320 \\
\hline Dementia, delirium and organic disorders & 11.972 & - & 1.466 & 8.17 & $<0.001$ & 9.099 to 14.845 \\
\hline Personality disorders & -4.747 & - & 1.266 & -3.75 & $<0.001$ & -7.228 to -2.265 \\
\hline Bipolar affective disorders & -4.215 & - & 1.814 & -2.32 & 0.02 & -7.772 to -0.657 \\
\hline Substance-related disorders & -3.499 & - & 0.993 & -3.52 & $<0.001$ & -5.444 to -1.553 \\
\hline Anxiety disorders & -1.545 & - & 1.032 & -1.5 & 0.134 & -3.568 to 0.478 \\
\hline Psychotic disorders & -0.401 & - & 1.16 & -0.35 & 0.73 & -2.675 to 1.873 \\
\hline Random effects & - & & & & & \\
\hline Individual & - & 426.975 & 15.096 & - & - & 398.389 to 457.612 \\
\hline Residual & - & 277.820 & 10.391 & - & - & 258.181 to 298.952 \\
\hline
\end{tabular}

psychosis/serious mental illness may be explained by higher detention rates in this patient group. Comparable with prospective studies and meta-analyses, we report associations between LOS and depression and anxiety. ${ }^{7,9}$ However, the current study made no distinction between conditions present before or developed during admission. It is possible LOS is affected by the onset of psychiatric symptoms and this warrants further investigation.

\section{Limitations}

Utilising patient records in an observational design precludes causative conclusions from this paper. Administrative data vary in completeness and consistency and in how well it reflects variables of interest but systematic review reports psychiatric diagnosis accuracy is moderate. ${ }^{31}$ Although frontline workers can be more accurate than clerical staff, quality varies between professionals. In this study, a large proportion of admissions did not have valid, categorisable psychiatric diagnoses. Conferring with liaison teams suggested data did not wholly reflect the clinical picture; self-harm numbers were especially low, raising questions about coding reliability. As self-harm occurs commonly in the context of psychiatric illness, it may be better accessed via clinical notes. Moreover, structured fields do not indicate symptom severity, which likely influences LOS. Similarly, without access to written records, it was difficult to control for multimorbidity; all diagnoses are not necessarily coded during every admission and it was difficult to determine if multiple diagnoses represented multimorbidity or changes to diagnosis. Finding a solution to this would be important given the impact multimorbidity may have on LOS. Challenges are compounded using longitudinal data because coding and staff changes occur over time. For this paper, not all expected A\&E liaison teams were available, which may have resulted from discrepancies in data extraction or changes to team names over time, although it is also possible the episodes extracted for $\mathrm{KCH}$ included both in-patient and A\&E referrals. This reflects limitations in the reliability of patient records as a data source for research.

Patient admissions are not linked across hospitals meaning LOS does not include continuation of stay between sites when patients are repatriated or potentially detained under the Mental Health Act. From a patient or national healthcare perspective, LOS across hospitals, including specialist mental health hospitals, may be of interest. Knowing the proportion of 'local' patients would also be useful as they may have longer LOS than those repatriated elsewhere while community follow-ups are arranged. This study did not compare the LOS of patients seen by liaison psychiatry with those with psychiatric diagnoses without liaison psychiatry contact; therefore, results cannot indicate the impact of liaison psychiatry on LOS, only which patients seen by liaison psychiatry experience the longest in-patient admissions and may be the most resource intensive. The authors only observed the overall effect of medical diagnoses on the association between psychiatric diagnoses and LOS - conclusions cannot therefore be drawn about the LOS or psychiatric comorbidity rate in patient groups with specific medical conditions, although this has been addressed in previous literature. ${ }^{32}$ Sociodemographic effects may be underestimated as there are statistical power issues in identifying inequalities at trust level. ${ }^{33}$

Finally, investment per admission is a proxy measure of liaison psychiatry resources and does not represent daily resources. Liaison investment does not reflect spend on local mental health provision including community-based services that provide the context of

\begin{tabular}{|c|c|c|c|c|c|c|}
\hline Fixed effects & Estimate & Variance & s.e. & Z-statistic & $P$ & $95 \% \mathrm{Cl}$ \\
\hline \multicolumn{7}{|l|}{ Fixed effects } \\
\hline \multicolumn{7}{|l|}{ Mental health diagnosis category ${ }^{a}$} \\
\hline Intercept $^{\mathrm{a}}$ & 18.719 & - & 3.342 & 5.60 & $<0.001$ & 12.168 to 25.269 \\
\hline Dementia, delirium and organic disorders & 7.149 & - & 1.356 & 5.27 & $<0.001$ & 4.491 to 9.807 \\
\hline Bipolar affective disorders & -5.008 & - & 1.652 & -3.03 & 0.002 & -8.247 to -1.769 \\
\hline Substance-related disorders & -2.806 & - & 0.926 & -3.03 & 0.002 & -4.621 to -0.992 \\
\hline Personality disorders & -2.078 & - & 1.163 & -1.79 & 0.074 & -4.358 to 0.202 \\
\hline Psychotic disorders & -1.792 & - & 1.085 & -1.65 & 0.099 & -3.919 to 0.335 \\
\hline Anxiety disorders & 0.786 & - & 0.958 & 0.82 & 0.041 & -1.091 to 2.664 \\
\hline \multicolumn{7}{|l|}{ Random effects } \\
\hline Individual & - & 294.524 & 12.195 & - & - & 271.566 to 319.422 \\
\hline Residual & - & 270.541 & 9.631 & - & - & 252.308 to 290.092 \\
\hline
\end{tabular}


Table 4 Table of marginal means for mental health diagnoses based on predictions of linear mixed-effects model

\begin{tabular}{|c|c|c|c|c|c|}
\hline $\begin{array}{l}\text { Mental health } \\
\text { diagnosis category }\end{array}$ & Mean & s.e. & $\begin{array}{l}\text { Z- } \\
\text { statistic }\end{array}$ & $P$ & $95 \% \mathrm{Cl}$ \\
\hline $\begin{array}{l}\text { Dementia, delirium } \\
\text { and organic }\end{array}$ & 23.00 & 1.33 & 17.28 & $<0.001$ & 20.39-25.61 \\
\hline Depressive disorders & 11.03 & 0.66 & 16.74 & $<0.001$ & $9.74-25.61$ \\
\hline $\begin{array}{l}\text { Psychotic disorders } \\
\text { and schizophrenia }\end{array}$ & 10.63 & 0.96 & 11.08 & $<0.001$ & $8.75-12.51$ \\
\hline Anxiety disorders & 9.48 & 0.82 & 11.59 & $<0.001$ & 7.88-11.09 \\
\hline $\begin{array}{l}\text { Substance-related } \\
\text { disorders }\end{array}$ & 7.53 & 0.76 & 9.71 & $<0.001$ & $6.01-9.05$ \\
\hline $\begin{array}{c}\text { Bipolar affective } \\
\text { disorders }\end{array}$ & 6.81 & 1.7 & 4.02 & $<0.001$ & $3.49-10.14$ \\
\hline Personality disorders & 6.28 & 1.1 & 6.9 & $<0.001$ & $4.12-8.45$ \\
\hline
\end{tabular}

liaison psychiatry and affect which patients present to hospital. Thus, although increases to investment per admission were associated with a small increase in LOS, this may, for example, reflect a shortage of available beds for onward referral and without liaison psychiatry, LOS may well be longer. As such this finding emphasises the importance of considering covariates in service evaluation. Furthermore, this study did not measure other consequences of liaison spending such as readmittance, waiting times or staff satisfaction. ${ }^{15}$

\section{Strengths}

This paper feeds into a widespread interest in the use of clinical records to improve services. Administrative data allows access to large and difficult-to-reach populations, provides representative samples not restricted by strict exclusion criteria, and uses realworld data less vulnerable to biases associated with controlled studies. Regarding methods, the longitudinal and naturalistic design reflects aims to explore services at a local level, allows temporal investigation and provides external validity to increase the utility of findings. Mixed-effects models acknowledged the inevitability of repeated measurements and the moderately large sample allowed for reasonable statistical power, reducing the likelihood of type 2 error.

\section{Implications}

With new Core24 implementation underway, the current study demonstrates how trust services can use patient data to execute national policy and meet local needs. Liaison psychiatry can improve LOS by reducing symptom burden, organising hospital transfers, facilitating discharge plans, increasing confidence of ward staff and signposting to community services. ${ }^{14}$ The observed changing rates of psychiatric diagnoses over time indicates that liaison services need to adapt training and recruitment regularly to capitalise on existing expertise and experience, and meet the needs of changing patient populations. Furthermore, as individual hospitals demonstrated different rates of diagnoses, our results suggest service changes need to be implemented on a team-byteam, rather than trust-wide, basis. Previous trust-wide evaluations of liaison psychiatry service expansions report improvements to LOS for dementia, delirium and severe mental illness. ${ }^{16}$ Specific findings from the current study could be used to guide resource allocation in SLaM services; for example, rotating doctors joining SLaM liaison psychiatry teams need to be well informed of local treatment options for depression, psychosis, organic disorders if the increased LOS associated with these disorders is to be reduced.

The Treat As One report found less than half (45.7\%) of hospitals have mandatory mental health training leading to poor recognition of psychiatric conditions among non-psychiatric clinicians and delays in discharge. ${ }^{34}$ Complex needs assessments, such as the Level
Of Care Utilisation System or INTERMED instrument, could be used to assess psychiatric input and assist discharge planning. ${ }^{35,36}$

The moderating effect of wider hospital and community contexts on liaison psychiatry performance must be emphasised. Since 2012, cumulative efficiency (savings) targets for SLaM services have reached over $21 \% .{ }^{37}$ Subsequent service changes have an impact on the complexity and severity of liaison psychiatry case-loads as liaison services absorb the consequences of amended communitybased services. To name a few, the national financial climate, government-set policy targets, availability of mental health beds, local general practice, community services and social care provision all must be considered when setting targets and evaluating liaison psychiatry. ${ }^{16}$ Furthermore, it is clear from our analysis that psychiatric diagnoses are one of many important factors contributing to LOS. Widespread change to service delivery is needed to tackle the impact and interaction between patient-level variables (i.e. physical and mental health, social deprivation) and service-level variables such as funding and community care availability.

Future research focusing on liaison psychiatry should consider further confounders such as risk, psychiatric comorbidity and treatment resistance. It would be pertinent to examine interactions between medical and psychiatric diagnoses to identify medical specialities requiring more familiarity with mental health. Additionally, the impact of demographic variables on mental health and LOS warrants further research given mixed findings on the effects of ethnicity. Research using administrative data requires balancing accuracy and maximising sample size. Sample size was lower than expected in this study and future studies should investigate alternative search terms to increase sample size. There is also an argument for linking LOS across hospital sites to elucidate psychiatric diagnoses' effect on total healthcare usage. The positive skew of LOS values by psychiatric diagnoses is worth comment; diagnostic groups with median LOS greater than 1 may indicate which are those more likely to receive an in-patient admission and future analysis may consider separate analysis of in-patient and A\&E episodes. Associations between psychiatric diagnoses and LOS are shown to differ between hospital trusts, ${ }^{16}$ suggesting a need for local evaluation prior to service changes.

In conclusion, this paper provides insight into the impact of psychiatric diagnoses on LOS in SLaM acute hospitals. Primary diagnoses of depression, dementia, delirium or organic disorders, psychotic disorders and schizophrenia are associated with longer LOS when controlling for physical health, demographic and hospital-level confounders. These findings underline the need for liaison psychiatry teams to adapt and reflect needs of individual hospital populations to effectively allocate resources and meet policy targets. Ongoing local-level evaluation is essential in helping liaison teams to provide expert care and education to non-psychiatric staff for the benefit of both patients and hospital performance.

Abbeygail Jones $\mathbb{D}$, MSc, Research Assistant, South London and Maudsley NHS Foundation Trust, UK; Helen Todman, MSC, NHS Programme Manager, South London and Maudsley NHS Foundation Trust, UK; Mujtaba Husain, Consultant Psychiatrist, South London and Maudsley NHS Foundation Trust, UK

Correspondence: Abbeygail Jones, Institute of Psychiatry, 16 De Cresigny Park, King's College London, London SE5 7AZ, UK. Email: abbeygail.jones@kcl.ac.uk

First received 31 Jan 2019, final revision 4 Aug 2019, accepted 22 Sep 2019

\section{Funding}

This research received no specific grant from any funding agency, commercial or not-for-profit sectors.

\section{Acknowledgements}

The authors would like to thank Jyoti and at the Biomedical Research Centre for helping with data extraction and Leo Koeser for advice during analysis. 


\section{Supplementary material}

Supplementary material is available online at https://doi.org/10.1192/bjo.2019.79.

\section{References}

1 Prince M, Patel V, Saxena S, Maj M, Maselko J, Phillips MR, et al. No health without mental health. Lancet 2007; 370: 859-77.

2 Sartorius N. Comorbidity of mental and physical disorders: a central challenge for medicine of the 21st century. Shanghai Arch Psychiatry 2013; 25: 68-9.

3 Rayner L, Matcham F, Hutton J, Stringer C, Dobson J, Steer S, et al. Embedding integrated mental health assessment and management in general hospital settings: feasibility, acceptability and the prevalence of common mental disorder. Gen Hosp Psychiatry 2014; 36: 318-24.

4 Thornicroft G. Physical health disparities and mental illness: the scandal of premature mortality. Br J Psychiatry 2011; 199: 441-2.

5 Lewis R, Edwards N. Improving Length of Stay: What can Hospitals do? Nuffield Trust, 2015

6 McManus S, Bebbington P, Jenkins R, Brugha T. Mental Health and Wellbeing in England: Adult Psychiatric Morbidity Survey 2014. NHS Digital, 2016.

7 Prina AM, Cosco TD, Dening T, Beekman A, Brayne C, Huisman M. The association between depressive symptoms in the community, non-psychiatric hospital admission and hospital outcomes: a systematic review. J Psychosom Res 2015; 78: 25-33.

8 Saravay SM, Steinberg MD, Weinschel B, Pollack S, Alovis N. Psychologica comorbidity and length of stay in the general hospital - ProQuest. Am J Psychiatry 1991; 148: 324-9.

9 Shoar S, Naderan M, Aghajani M, Sahimi-Izadian E, Hosseini-Araghi N, Khorgami Z. Prevalence and determinants of depression and anxiety symptoms in surgical patients. Oman Med J 2016; 31: 176-81.

10 Wancata J, Benda N, Windhaber J, Nowotny M. Does psychiatric comorbidity increase the length of stay in general hospitals? Gen Hosp Psychiatry 2001; 23: $8-14$.

11 Rosman M, Rachminov O, Segal O, Segal G. Prolonged patients' In-Hospital Waiting Period after discharge eligibility is associated with increased risk of infection, morbidity and mortality: a retrospective cohort analysis. BMC Health Serv Res 2015; 15: 246.

12 Muakkassa FF, Marley RA, Billue KL, Marley M, Horattas S, Yetmar Z, et al. Effect of hospital length of stay on functional independence measure score in trauma patients. Am J Phys Med Rehabil 2016; 95: 597-607.

13 National Institute for Health and Care Excellence. Emergency and Acute Medical Care in Over 16s: Service Delivery and Organisation. NICE Guideline NG94. NICE, 2018

14 National Institute for Health and Care Excellence. Achieving Better Access to 24/7 Urgent and Emergency Mental Health Care - Part 2: Implementing the Evidence-based Treatment Pathway for Urgent and Emergency Liaison Mental Health Services for Adults and Older Adults. NICE, 2016.

15 Tadros G. Intelligent outcome measures in liaison psychiatry: essential even if not desirable. BJPsych Bull 2016; 40: 195-8.

16 Becker L, Saunders R, Hardy R, Pilling S. The RAID Model of Liaison Psychiatry Report on the Evaluation of Four Pilot Services in east London. UCL Partners, 2016 (https://www.ucl.ac.uk/pals/sites/pals/files/uclp_raid_evaluation_report.pdf).

17 NHS England. Five Year Forward View. NHS England, 2014

18 Dorning H, Davies A, Blunt I. Focus on: People with Mental III Health and Hospital Use. Exploring Disparities in Hospital Use for Physical Healthcare. Nuffield Trust and the Health Foundation, 2015 (https://www.nuffieldtrust. org.uk/files/2018-10/1540147713_qualitywatch-mental-ill-health-and-hospital-use-summary.pdf)

19 House A, Smith C, Hulme C, Tringwell P, Fossey M, Murray CC, et al. Liaison Psychiatry: Measurement And Evaluation of Service Types, Referral patterns and Outcomes LP-MAESTRO. National Institute for Health Research, 2015.

20 Perera G, Broadbent M, Callard F, Chang C-K, Downs J, Dutta R, et al. Cohort profile of the South London and Maudsley NHS Foundation Trust Biomedical Research Centre (SLaM BRC) Case Register: current status and recent enhancement of an Electronic Mental Health Record-derived data resource. BMJ Open 2016; 6: e008721.

21 Department of Health. Discharging Older Patients from Hospital - National Audit Office (NAO) Report. National Audit Office, 2016.

22 Motzek $\mathrm{T}$, Junge $\mathrm{M}$, Marquardt $\mathrm{G}$. Impact of dementia on length of stay and costs in acute care hospitals. Rom J Gerontol Geriatr 2017; 50: 59-66.

23 World Health Organization. International Classification of Diseases 10th Revision (ICD-10). WHO, 2010

24 Haile A, Carter C. CDS Type 011 - Emergency Care Data Set (ECDS) User Guidance. Health and Social Care Information Centre, 2017.

25 StataCorp. Stata Statistical Software: Release 12. StataCorp LP, 2011.

26 StataCorp. Stata Statistical Software: Release 13. StataCorp LP, 2013.

27 Laird N, Ware J. Random-effects models for longitudinal data. Biometrics 1982; 38: 963-74.

28 Grafen A, Hails R. Modern Statistics for the life sciences. Oxford University Press, 2002.

29 Meier K, Brudney J, Bohte J. Applied Statistics for Public and Nonprofit Administration. Cengage Learning, 2014.

30 Harrison XA, Donaldson L, Correa-Cano ME, Evans J, Fisher DN, Goodwin C et al. A brief introduction to mixed effects modelling and multi-model inference in ecology. PeerJ 2018; 6: e4794.

31 Davis KAS, Sudlow CLM, Hotopf M. Can mental health diagnoses in administrative data be used for research? A systematic review of the accuracy of routinely collected diagnoses. BMC Psychiatry 2016; 16: 263.

32 Bradford AL, Crider CC, Xu X, Naqvi SH. Predictors of recurrent hospital admission for patients presenting with diabetic ketoacidosis and hyperglycemic hyperosmolar state. J Clin Med Res 2017; 9: 35-9.

33 Langford $\mathrm{KM}$, Bottle $\mathrm{A}$, Aylin $\mathrm{PP}$, Ward $\mathrm{H}$. Using routine data to monitor inequalities in an acute trust: a retrospective study. BMC Health Serv Res 2012; 12 104

34 Cross S, Srivastava V, Shotton H, Butt A, Protopapa K, Mason M. Treat As One. Bridging the Gap Between Mental and Physical Healthare in General Hospitals. NCEPOD, 2017 (https://www.ncepod.org.uk/2017report1/downloads/ TreatAsOne FullReport.pdf).

35 American Psychiatric Association. LOCUS Level of Care Utilization System for Psychiatric and Addictions Services, Adult Version 2010. APA, 2009.

36 Huyse FJ, Lyons JS, Stiefel FC, Slaets JPJ, de Jonge P, Fink P, et al. 'INTERMED': a method to assess health service needs. Gen Hosp Psychiatry 1999; 21: 39-48.

37 SLaM NHS Foundation Trust. Annual Report and Summary Accounts 2017/2018 (press release). SLaM NHS Foundation Trust, 2018.

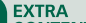

